\title{
Transdifferentiation during Heart Regeneration
}

\author{
Xianmei Meng ${ }^{1,2 *}$, Ali Nsair ${ }^{1,2 *}$ \\ ${ }^{1}$ Cardiovascular Research Laboratories, Department of Medicine, David Geffen School of Medicine, University of California Los Angeles, Los Angeles, CA 90095, USA \\ ${ }^{2}$ The Edythe and Eli Broad Center for Regenerative Medicine and Stem Cell Research, David Geffen School of Medicine, University of California Los Angeles, Los \\ Angeles, CA 90095, USA
}

\begin{abstract}
Heart disease is the leading cause of mortality worldwide. Transdifferentiation, also called direct reprogramming, is a process where a mature somatic cell transforms into another distinct cell type. Rapidly accumulating studies have suggested that transdifferentiation of mature somatic cells into cardiomyocytes and other cell types has provided tremendous hope for the treatment of heart disease. Here I summarize the recent advances, specifically regarding the strategies used for transdifferentiation of cardiac cells during heart regeneration.
\end{abstract}

Keywords: Transdifferentiation; Heart disease; Cardiomyocyte; Heart regeneration

\section{Introduction}

Heart attack or myocardial infarction (MI), the largest risk factor for heart failure, can result in the loss of $25 \%$ of the myocardial mass of the heart [1]. Although postnatal cardiomyocytes retain the capabilities of proliferation and self-renewal, adult cardiomyocytes fail to re-enter the cell cycle to replenish damaged tissue, and adult heart has limited endogenous regenerative capacity [2].

Transdifferentiation is a process where one cell type converts into another without undergoing an intermediate state of pluripotency or progenitor. Transdifferentiation has caused great excitement in regenerative medicine since it has some main advantages such as shorter times required, less chance of tumor formation, and the avoidance of ethical issues. Thus, it can be anticipated that transdifferentiation of mature somatic cells into cardiomyocytes will open up new possibilities for disease modeling and cell therapy for cardiovascular disease. Here, I review the recent advances in transdifferentiation of cardiac cells during heart regeneration.

\section{Transcription Factor-Induced Cardiac Transdifferen- tiation}

In 1987, Davis et al first demonstrated that mouse fibroblasts were directly reprogrammed to myoblasts with high efficiency using the transcription factor $M y o D$ [3]. The finding rocked the world and made transdifferentiation an interesting and promising idea for regenerative medicine. However, due to the fact that it was difficult to find the master gene(s) for cardiac muscle, the interest in transdifferentiation of cardiac cells waned. Spurred by the discovery of induced pluripotent stem cells (iPSCs) [4,5], the investigators have now steered their blueprint back to transdifferentiation for cardiac cells to treat cardiovascular disease. Efe et al. [6] demonstrated that mouse embryonic fibroblasts (MEFs) can be directly reprogrammed to contracting cardiomyocytes in a fast and efficient manner by shortcutting the conventional reprogramming towards pluripotency through overexpression of Oct4, Sox2, Klf4 and $c-M y c$. This work has potentially widened the implications for iPSCfactor-based reprogramming and extended the existing paradigm.

Current studies of cardiac development have identified a number of transcription factors that are essential for transdifferentiation of cardiac cells. In 2009, Takeuchi and Bruneau claimed that overexpression of the chromatin-remodeling factor Baf60c, Gata4, and $T b \times 5$ was sufficient to reprogram noncardiogenic mesoderm into beating cardiomyocytes by a mechanism involving the induction of $N k x 2.5$ by Gata4 and Baf60c [7]. Cardiac fibroblasts comprise over $50 \%$ of all the cells in the heart and can replace the lost cardiomyocytes when a scar is formed in the site of injury [1]. Thus, cardiac fibroblasts in situ can serve as a good source of cardiomyocytes to treat heart disease. In 2010, Ieda and colleagues first started the journey to transdifferentiate mouse cardiac fibroblasts into cardiomyocytes [8]. They winnowed 14 cardiac regulatory genes to 3 transcription factors Gata4, Mef2c, and Tbx5 (GMT) that made an efficient cocktail to transdifferentiate mouse cardiac fibroblasts into cardiomyocytes in vitro and in vivo [8]. However, the effectiveness of the GMT combination was challenged by the study performed by Chen et al [9]. Chen et al. [9] claimed that GMT overexpression in mouse fibroblasts was inefficient to transdifferentiate the cells to mature cardiomyocytes. The in vivo experiments further indicated poor cell survival and engraftment of these induced mouse cardiac fibroblasts into injured mouse hearts with minimal induction of cardiomyocyte genes [9]. The cause of the discrepancy between the two studies may be due to the subtle differences between their experimental procedures such as titers of lentiviruses used to deliver GMT, the substrates used to plate cells or the cell densities. Probably the main reason is the developmental stage of cardiac fibroblasts used for transdifferentiation. Compared to neonatal cardiac fibroblasts used in most of Ieda's experiments, the cardiac fibroblasts used in Chen's study were from 3-6 weeks old mice [9]. Previous studies showed that cells isolated from embryos and neonates retain more plasticity than adult cells, and thus represent the ideal cell type for feasibility experiments [7].

*Corresponding authors: Xianmei Meng, Cardiovascular Research Laboratories, Department of Medicine and The Edythe and Eli Broad Center for Regenerative Medicine and Stem Cell Research, David Geffen School of Medicine, University of California Los Angeles, Los Angeles, CA 90095, USA, Tel: 310-825-5635; E-mail: xmeng@mednet.ucla.edu

Ali Nsair, Cardiovascular Research Laboratories, Department of Medicine and The Edythe and Eli Broad Center for Regenerative Medicine and Stem Cell Research, David Geffen School of Medicine, University of California Los Angeles, Los Angeles, CA 90095, USA, Tel: 310-825-5635; E-mail: ansair@mednet.ucla.edu

Received March 12, 2014; Accepted April 02, 2014; Published April 04, 2014

Citation: Meng X, Nsair A (2014) Transdifferentiation during Heart Regeneration. J Stem Cell Res Ther 4: 188. doi:10.4172/2157-7633.1000188

Copyright: ( $) 2014$ Meng X, et al. This is an open-access article distributed under the terms of the Creative Commons Attribution License, which permits unrestricted use, distribution, and reproduction in any medium, provided the original author and source are credited. 
Science develops along with discrepancy and renovation. Song et al demonstrated that the addition of Hand2 to GMT increased the efficiency of cardiac reprogramming in vitro and in vivo directly converted nonmyocyte to myocytes [10]. These myocytes possess similar characteristics to the endogenous cardiomyocytes such as cardiac gene expression, sarcomere structure, and electrophysiological features [10]. To promote the survival of viral-transduced cells and improve the reprogramming efficiency of GMT in vivo, Inagawa et al generated a polycistronic retrovirus expressing GMT at nearequimolar levels using self-cleaving $2 \mathrm{~A}$ peptides and induced cardiomyocyte-like cells in infarct heart in immunosuppressed nude mice [11]. By directly injecting GMT retroviruses into mouse infarcted hearts, Qian et al. [12] demonstrated that resident cardiac fibroblasts generated approximately $35 \%$ of cardiomyocytes in the border/infarct zone and half of these newly induced cardiomyocyte-like cells showed well-organized sarcomeric structures and functional characteristics. These results indicated that in vivo reprogramming induces mature cardiomyocyte-like cells more efficiently than in vitro reprogramming [12]. Protze et al. [13] screened 120 triplet combinations of 10 important developmental cardiac transcription factors expressed via lentiviruses to test their ability to induce MEFs into a myocyte-like phenotype. They demonstrated that $T b x 5, M e f 2 c$, and Myocd led to a broader spectrum of cardiac gene expression compared to GMT [13]. These findings may offer a new regenerative strategy that the abundant fibroblasts in the heart can be transdifferentiated into new cardiomyocyte-like cells in vitro and in vivo and thus replace cardiomyocytes lost in injured hearts.

It is of enormous clinical significance to translate the knowledge gained in the mouse system into human cells. However, human cells are more resistant to transdifferentiation than mouse cells. Using the similar combinations of GMT, researchers failed to transdifferentiate human fibroblasts to cardiomyocyte-like cells even though these combinations could push human fibroblasts into a cardiomyocytelike state in vitro [14-16]. Nam and colleagues demonstrated that the combination of 4 transcription factors GATA4, HAND2, TBX5, and MYOCD plus microRNAs (miRNAs) only converted $\sim 20 \%$ of human fibroblasts into a cardiomyocyte-like state with rare beating cells even after a long period in culture [14]. Although Fu et al disclosed that the combination of 7 transcription factors GATA4, MEF2C, TBX5, ESRRG (a nuclear hormone receptor), MESP1, MYOCD and ZEPM2 upregulated hundreds of cardiomyocyte-enriched genes and downregulated hundreds of fibroblast-enriched transcripts in vitro, most of the cells were only partially transdifferentiated without visible contractile activity [15]. Wada et al showed that human fibroblasts induced by the combination of 5 transcription factors GATA4, MEF2C, TBX5, MESP1, and $M Y O C D$ changed the cell morphology from a spindle shape to a rod-like or polygonal shape, along with cardiac gene expression and exhibition of electrophysiological features when the induced immature cardiomyocyte-like cells matured [16]. Despite substantial progress recently made in human cells, the different set of reprogramming factors used to convert human fibroblasts to cardiomyocytes may mark a different protocol in human cells compared to mouse system. Thus, larger animal models using pigs or monkeys should be considered to bridge in vivo reprogramming results from mouse system to the clinic.

To this end, this approach was the first to demonstrate large-scale generation of new cardiomyocytes following MI as well as substantial improvement in heart function in mouse system. Moreover, they represent proof-of-concept for a new clinical paradigm in repairing the heart following MI and preventing heart failure by exploiting endogenous scar tissue. However, this strategy still has a long way to go to be translated to the clinical use because overexpression of transcription factors to achieve reprogramming obviously represents the risk of genomic integration, insertional mutagenesis, potential "leakage" into non-targeted tissue, and cardiac arrhythmias after transplantation to the human heart.

\section{Micro RNA-Induced Cardiac Transdifferentiation}

MiRNAs, a class of functional noncoding RNAs consisting of $\sim 22$ nucleotides, regulate gene expression and play a critical role in cardiomyogenesis [17]. Jayawardena et al showed that a combination of miRNAs 1, 133, 208, and 499 was sufficient to transdifferentiate mouse fibroblasts into cardiomyocyte-like cells in vitro and in vivo [18]. Notably, miR-1 alone was capable to fulfill this process with a Janus kinase (JAK)-1 inhibitor [18]. Nam and colleagues demonstrated that the two miRNAs 1 and 133 further improved myocardial conversion of human fibroblasts induced by the combination of 4 transcription factors GATA4, HAND2, TBX5, and MYOCD and eliminated the need of MEF2C [14]. These studies have revealed that miRNAs are promising tools for transdifferentiation.

\section{Small Chemical-Induced Cardiac Transdifferentiation}

Considerable evidence suggests that small molecules or chemicals, such as DNA methyltransferase inhibitors, histone methyltransferase inhibitors, histone deacetylase inhibitors, glycogen synthase kinase-3 beta (GSK-3 $\beta$ ) inhibitors, manipulating epigenetic status and signaling pathways can enhance reprogramming and functionally replace some reprogramming factors [19-22]. The studies performed by Efe and Jayawardena et al also included JAK-1 inhibitor to increase the transdifferentiation efficiency by suppressing pluripotencypromoting pathways $[6,18]$. Thal et al. [23] revealed that mouse and human endothelial progenitor cells were transdifferentiated into cardiomyocytes using inhibitors 5-Azacytidine for DNA methyltransferases, valproic acid for histone deacetylases and BIX01294 for G9a histone dimethyltransferases. Our unpublished data have shown that small molecules alone can directly reprogram human cardiac fibroblasts into cardiomyocyte-like cells. Notably, we have successfully achieved up to 100 -fold upregulation of cardiomyocyte structural genes following small molecule treatment. These findings could help develop a comprehensive therapeutic modality to revolutionize the treatment of MI and heart failure. The strategy only using small molecules would provide for the possibility of eventual targeted delivery of the reprogramming cocktail via a catheterdelivered epicardial patch, thereby avoiding an open-chest procedure and potentially could be performed in an outpatient setting.

\section{Coculture-Induced Cardiac Transdifferentiation}

Recent studies have indicated that cell-to-cell contact but not cellular fusion mediated the microenvironment factors, which made it possible to transdifferentiate adult stem/progenitor cells into cardiomyocytes [24-26]. Yoon et al demonstrated that rat mesenchymal stem cells cocultured with rat neonatal cardiomyocytes for 5 days were capable to transdiffererentiate into cardiomyocytes [24]. Badorff et al. [25] claimed that endothelial progenitor cells derived from human peripheral blood mononuclear cells cocultured with rat cardiomyocytes were transdifferentiated into cardiomyocytes. Human amniotic membrane-derived mesenchymal cells cocultured with murine fetal cardiomyocytes were transdifferentiated into cardiomyocytes in vitro and in vivo [26]. These studies reasoned that coculture could provide a safer and more clinical relevant cardiomycytes.

As stated above, transdifferentiation has already brought a great 
hope for the treatment for heart diseases. However, there are still some challenges including meticulous studies in human cells to bridge the gap from mouse system to human cells, understanding the mechanisms involved in transdifferentiation, improving the in vivo efficiency and efficacy in small and large animal models. Continued advances in transdifferentiation could fully explore small molecules that probably own the potential to replace lost cardiomyocytes via in vivo transdifferentiation without cell transplantation. The eventual targeted delivery of the reprogramming cocktail only consisting of small molecules via a catheter-delivered epicardial patch directly to the injured heart to induce regeneration in situ will avoid many of the pitfalls of cell-based therapies including cell type and number to be used, route of administration and retention and survival of transplanted cells, and possible immune rejection. Hopefully, the technology of cardiac transdifferentiation could help patients with heart disease in the near future.

\section{Acknowledgements}

The author is very grateful to Dr. Ben Van Handel and Dr. Bo Li for their valuable discussion and comments.

\section{References}

1. Go AS, Mozaffarian D, Roger VL, Benjamin EJ, Ford ES, et al. (2014) Heart Disease and Stroke Statistics-2014 Update: A Report From the American Heart Association. Circulation 129(3): e28-e292.[PubMed]

2. Senyo SE, Steinhauser ML, Pizzimenti CL, Yang VK, Cai L, et al. (2013) Mammalian heart renewal by pre-existing cardiomyocytes. Nature 493(7432): 433-436.[PubMed]

3. Davis RL, Weintraub $H$, Lassar AB (1987) Expression of a single transfected cDNA converts fibroblasts to myoblasts. Cell 51(6): 987-1000.[PubMed]

4. Takahashi K, Yamanaka S (2006) Induction of pluripotent stem cells from mouse embryonic and adult fibroblast cultures by defined factors. Cell 126(4): 663-676.[PubMed]

5. Takahashi K, Tanabe K, Ohnuki M, Narita M, Ichisaka T, et al. (2007) Induction of pluripotent stem cells from adult human fibroblasts by defined factors. Cell 131(5): 861-872.[PubMed]

6. Efe JA, Hilcove S, Kim J, Zhou H, Ouyang K, et al. (2011) Conversion of mouse fibroblasts into cardiomyocytes using a direct reprogramming strategy. Nat Cell Biol 13(3): 215-222.[PubMed]

7. Takeuchi JK, Bruneau BG (2009) Directed transdifferentiation of mouse mesoderm to heart tissue by defined factors. Nature 459(7247): 708-711. [PubMed]

8. leda M, Fu JD, Delgado-Olguin P, Vedantham V, Hayashi Y, et al. (2010) Direct reprogramming of fibroblasts into functional cardiomyocytes by defined factors. Cell 142(3): 375-386.[PubMed]

9. Chen JX, Krane M, Deutsch MA, Wang L, Rav-Acha M, et al. (2012) Inefficient reprogramming of fibroblasts into cardiomyocytes using Gata4, Mef2c, and Tbx5. Circ Res 111(1): 50-55.[PubMed]

10. Song K, Nam YJ, Luo X, Qi X, Tan W, et al. (2012) Heart repair by reprogramming non-myocytes with cardiac transcription factors. Nature 485(7400): 599-604. [PubMed]

11. Inagawa K, Miyamoto K, Yamakawa H, Muraoka N, Sadahiro T, et al. (2012) Induction of cardiomyocyte-like cells in infarct hearts by gene transfer of Gata4, Mef2c, and Tbx5. Circ Res 111(9): 1147-1156.[PubMed]

12. Qian L, Huang Y, Spencer Cl, Foley A, Vedantham V, et al. (2012) In vivo reprogramming of murine cardiac fibroblasts into induced cardiomyocytes. Nature 485(7400): 593-598.[PubMed]
13. Protze S, Khattak S, Poulet C, Lindemann D, Tanaka EM, et al. (2012) A new approach to transcription factor screening for reprogramming of fibroblasts to cardiomyocyte-like cells. J Mol Cell Cardiol 53(3): 323-332.[PubMed]

14. Nam YJ, Song K, Luo X, Daniel E, Lambeth K, et al. (2013) Reprogramming of human fibroblasts toward a cardiac fate. Proc Natl Acad Sci USA 110(14): 5588-5593.[PubMed]

15. Fu JD, Stone NR, Liu L, Spencer Cl, Qian L, et al. (2013) Direct Reprogramming of Human Fibroblasts toward a Cardiomyocyte-like State. Stem Cell Reports 1(3): 235-247.[PubMed]

16. Wada R, Muraoka N, Inagawa K, Yamakawa H, Miyamoto K, et al. (2013) Induction of human cardiomyocyte-like cells from fibroblasts by defined factors. Proc Natl Acad Sci USA 110(31): 12667-12672.[PubMed]

17. Liu N, Olson EN (2010) MicroRNA regulatory networks in cardiovascular development. Dev Cell 18(4): 510-525.[PubMed]

18. Jayawardena TM, Egemnazarov B, Finch EA, Zhang L, Payne JA, et al (2012) MicroRNA-mediated in vitro and in vivo direct reprogramming of cardiac fibroblasts to cardiomyocytes. Circ Res 110(11): 1465-1473.[PubMed]

19. Zhu S, Li W, Zhou H, Wei W, Ambasudhan R, et al. (2010) Reprogramming of human primary somatic cells by OCT4 and chemical compounds. Cell Stem Cell 7(6): 651-655.[PubMed]

20. Meng X, Neises A, Su RJ, Payne KJ, Ritter L, et al. (2012) Efficient reprogramming of human cord blood CD34+ cells into induced pluripotent stem cells with OCT4 and SOX2 alone. Mol Ther 20(2): 408-416.[PubMed]

21. Meng X, Su RJ, Baylink DJ, Neises A, Kiroyan JB, et al. (2013) Rapid and efficient reprogramming of human fetal and adult blood CD34+ cells into mesenchymal stem cells with a single factor. Cell Res 23(5): 658-672.[PubMed]

22. Hou P, Li Y, Zhang X, Liu C, Guan J, et al. (2013) Pluripotent stem cells induced from mouse somatic cells by small-molecule compounds. Science 341(6146): 651-654.[PubMed]

23. Thal MA, Krishnamurthy P, Mackie AR, Hoxha E, Lambers E, et al. (2012) Enhanced Angiogenic and Cardiomyocyte Differentiation Capacity of Epigenetically Reprogrammed Mouse and Human Endothelial Progenitor Cells Augments Their Efficacy for Ischemic Myocardial Repair. Circ Res 111(2) 180-190.[PubMed]

24. Yoon J, Shim WJ, Ro YM, Lim DS (2005) Transdifferentiation of mesenchymal stem cells into cardiomyocytes by direct cell-to-cell contact with neonatal cardiomyocyte but not adult cardiomyocytes. Ann Hematol 84(11): 715-721. [PubMed]

25. Badorff C, Brandes RP, Popp R, Rupp S, Urbich C, et al. (2003) Transdifferentiation of blood-derived human adult endothelial progenitor cells into functionally active cardiomyocytes. Circulation 107(7): 1024-1032. [PubMed]

26. Tsuji H, Miyoshi S, Ikegami Y, Hida N, Asada H, et al. (2010) Xenografted human amniotic membrane-derived mesenchymal stem cells are immunologically tolerated and transdifferentiated into cardiomyocytes. Circ Res 106(10): 16131623.[PubMed] 